\title{
MTDH Mediates Estrogen-Independent Growth and Tamoxifen Resistance by Down-Regulating PTEN in MCF-7 Breast Cancer Cells
}

\author{
Chunyuan Xu $\mathrm{u}^{\mathrm{a}, \mathrm{b}}$ Xiaoli Kong ${ }^{\mathrm{c}}$ Haiji Wang ${ }^{\mathrm{a}, \mathrm{d}}$ Ning Zhang ${ }^{\mathrm{c}}$ Xiangnan Kong ${ }^{\mathrm{c}}$ \\ Xia Ding ${ }^{a}$ Xiaoyan Lic Qifeng Yang ${ }^{c}$
}

aDepartment of Oncology, Qilu Hospital, Shandong University, Jinan, bepartment of Oncology, Qingdao Municipal Hospital, Qingdao, 'Department of Breast Surgery, Qilu Hospital, Shandong University, Wenhua, Jinan, dDepartment of Oncology, Affiliated Hospital of Qingdao University Medical College, Qingdao, P.R. China

\section{Key Words}

MTDH・Estrogen-independent growth • Tamoxifen resistance $•$ PTEN • Breast cancer

\begin{abstract}
Background: About $70 \%$ of human breast cancers express estrogen receptor $\alpha(E R \alpha)$ and in this kind of breast cancer estrogen plays an important role. Estrogen independent growth has been reported to promote resistance to one of the selective estrogen receptor modulators (SERMs) tamoxifen which is clinically the first line treatment for patients with ER $\alpha$-positive breast cancer. The resistance of tamoxifen is a major problem in the clinical management of breast cancer. Methods: We used MCF-7 cells with ectopic expression of MDTH in this study. MTT, clone formation and tumor formation in nude mice methods were utilized to confirm the role of MTDH in estrogen-independent growth and tamoxifen resistance. Flow cytometry, western blot and siRNA were used to study the detailed mechanisms. Results: We found that MTDH could mediate estrogen-independent growth and induce resistance to tamoxifen in ER $\alpha$-positive breast cancer cells. MTDH could reduce the expression of PTEN, up-regulate AKT and BCL2 and inhibit the apoptosis induced by tamoxifen. Conclusion: Our study indicated that MTDH was a candidate marker to predict the clinical efficacy of tamoxifen and targeting MTDH would overcome the resistance to tamoxifen in breast cancer cells.
\end{abstract}




\section{Introduction}

Breast cancer is the most common malignant tumor diagnosed in women worldwide and has become leading cause of cancer death in economically developing countries [1]. About $70 \%$ of breast cancers express high concentration of estrogen receptor (ER $\alpha$ ) and candidates for endocrine therapy. Over the past few decades, tamoxifen, a selective ER modulator, has been the most commonly prescribed in endocrine therapy of ER $\alpha$ positive breast cancer and has been recommended as a preventative drug for female at high risk of developing breast cancer [2]. Tamoxifen which was used as an adjuvant drug has significantly improved the survival of early stage breast cancer patients. Nevertheless, nearly $35 \%$ of breast cancer patients fail to adjuvant tamoxifen therapy [3]. Tamoxifen resistance has been a major problem in the endocrine therapy of breast cancer. A large number of patients exhibit de novo tamoxifen resistance despite the presence of ER $\alpha$ in their breast tumors. Unfortunately, the molecular mechanisms of such resistance remain a poor understanding and serious clinical problems. It was considered that molecular crosstalk between ER and various growth factor signaling pathways might play important role in resistance to tamoxifen [4]. And a recent study reported that miR-375 and its target gene MTDH also worked in mechanisms of resistance to tamoxifen [5]. These studies provided research directions for understanding of resistance to tamoxifen. However it is still far from enough.

MTDH (metadherin, also known as AEG-1 and Lyric) is a novel oncogene found in 2002 [6]. Our previously studies show that it is widely over-expressed in many breast cancer cell lines or tumors and plays a crucial role in tumor invasion, metastasis and drug resistance [7-10]. Nearly 50\% ER-positive breast cancer patient express high concentration of MTDH by immunohistochemicial method and was significantly correlated with an aggressive phenotype and a poor prognosis [11]. In many ER-positive breast cancer cells estrogen $\left(E_{2}\right)$ plays a crucial role [12]. But it hasn't been reported whether $E_{2}$ is still important in tumor proliferation and progression in MTDH over-expressed ER-positive breast cancers. Otherwise the potential efficacy of MTDH in the treatment of tamoxifen in breast cancer has been unknown. In this study we over-expressed MTDH in ER-positive breast cancer cell line MCF-7 and found that MTDH could promote estrogen independent growth in MCF-7 cell line and further induce tamoxifen-resistance through inhibiting apoptosis by regulating PTEN/ AKT pathway.

\section{Materials and Methods}

\section{Reagents}

The 17- $\beta$ estradiol was purchased from Wako Pure Chemical Industries, Ltd. (Osaka, Japan), dissolved to a final concentration of $1 \mathrm{mM}$ and stored at $-20^{\circ} \mathrm{C}$. Tamoxifen was purchased from Sigma-Aldrich (St. Louis, MO, USA). Estradiol pellet was purchased from Innovative Research of America. Rabbit polyclone antibody against MTDH was obtained from Invitrogen (Carlsbad, CA, USA). Antibodies against ER $\alpha$, BCL-2 were purchased from Dako (Glostrup, Denmark). Rabbit antibodies against PTEN, p-AKT(Ser473), GAPDH and mouse anti-AKT were purchased from Cell Signaling Technology (Beverly, MA, USA). Mouse monoclone antibody against $\beta$-actin was purchased from Sigma-Aldrich (St. Louis, MO, USA). The HRP-labeled secondary antibodies were from KPL (Gaithersburg, MD, USA).

\section{Cell culture}

The ER $\alpha$ negative cell lines MDA-MB-231, MDA-MB-468 and ER $\alpha$ positive cell lines MCF-7 and T47D were obtained from the American Type Culture Collection (Rockville, MA, USA) and grown in Dulbecco's Modified Eagle's Medium(DMEM, Gibco-BRL, Rockville, IN, USA) with 10\% fetal bovine serum (FBS, Haoyang biological manufacture Co. Ltd, Tianjin, China), 100U/ml penicillin and $100 \mu \mathrm{g} / \mathrm{ml}$ streptomycin at $37^{\circ} \mathrm{C} 5 \% \mathrm{CO} 2$ incubator. And $17 \beta$-estradiol ( $\mathrm{E}_{2}$; Sigma-Aldrich, St. Louis, MO, USA) treatment cells were plated with phenol red-free DMEM medium supplemented with 5\% charcoal-stripped FBS (Biochrom AG, Berlin, Germany) with or without $\mathrm{E}_{2}$. 


\begin{tabular}{|c|c|c|}
\hline Cellular Physiology & Cell Physiol Biochem 2014;33:1557-1567 & \\
\hline and Biochemistry & 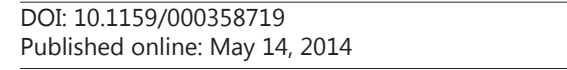 & $\begin{array}{l}\text { Q } 2014 \text { S. Karger AG, Basel } \\
\text { www.karger.com/cpb }\end{array}$ \\
\hline
\end{tabular}

Vector construction and identification

The vector construction was performed as described previously [8]. Briefly, the cDNA representing the complete open reading frame of MTDH was cloned into the BamHI-Xhol vector fragment derived from pcDNA3.1/myc-His A vector (3.1n) (Invitrogen, USA) to generate pcDNA3.1-MTDH (3.1 m). The expression plasmids were verified by sequencing of both strands.

\section{Transfection and expression of MTDH}

We transfected MCF-7 cells by using Lipofectamine TM 2000 (Invitrogen, Karlsruhe, Germany) according to the manufacturer's instructions. The cells were seeded into 24-well culture plates (Biochrom AG, Berlin, Germany) at a density of $3 \times 10^{4}$ cells/well. The next day, cells were washed twice with PBS. Transfection mixes were added and cells were incubated for $4 \mathrm{~h}$ at $37^{\circ} \mathrm{C}$ and $5 \% \mathrm{CO} 2$. Subsequently, the transfection medium was replaced by complete medium. Cells were passaged at a 1:10 dilution into fresh growth medium $24 \mathrm{~h}$ after transfection and the following day G418 was added at the concentration of $600 \mathrm{ug} / \mathrm{ml}$ for selecting the stable cell lines. The selected cells were maintained in DMEM with $300 \mu \mathrm{g} / \mathrm{ml}$ G418. The efficiency of over-expression of MTDH in this cell line was tested by western blot analysis and real-time PCR (RT-PCR). Empty 3.1n vector was used as control. The stable selected cells transfected with 3.1n was called MCF7-3.1n while cells with $3.1 \mathrm{~m}$ called MCF7-3.1m.

\section{Real-time RT-PCR}

Total RNA was isolated using Trizol (Invitrogen Corporate, Carlsbad, CA, USA) according to the manufacturer's protocol. RNAs werereverse transcribed by using PrimeScript ${ }^{\circledR}$ RT reagentKit(Takara,Dalian, China). The resulting cDNA was used for quantitative real-time PCR on a StepOne Plus instrument (Applied Biosystems, Carlsbad, CA, USA) with SYBR green (Takara, Dalian, China) detection. Primers involved were the MTDH primers forward '-AAATAGCCAGCCTATCAAGACTC-3', reverse 5'-TTCAGACTTGGTCTGTGAAGGAG-3') and the GAPDH primers (forward 5'-GGGCTGCTTTTAACTCTGGTAAAG-3', reverse 5'-CCATGGGTGGAATCATATTGG-3'). StepOne Plus software was used to calculate crossing threshold (Ct) points from the fluorescence curves and the delta/delta-Ct method was applied to quantify the induction of mRNA as compared to the control sample.

\section{Cell proliferation assay}

The cell proliferation with or without $\mathrm{E}_{2}$ and drug sensitivity to tamoxifen was measured by 3-(4,5-dimethylthiazol-2-yl)-2,5- diphenyltetrazolium bromide (MTT, Amresco, Solon, OH, USA). For estrogen dependent assay, cells were plated in 96-well culture plates at the concentration of 2000 cells/ well. The next day, different concentrations of $\mathrm{E}_{2}$ were added into $100 \mathrm{ul}$ dilution of the culture medium. Cells were incubated for 96 hours at $37^{\circ} \mathrm{C}, 5 \% \mathrm{CO}_{2}$. For estrogen independent growth, 2000 cells/well were plated in 96 -well culture plates in phenol red-free DMEM medium supplemented with $5 \%$ charcoalstripped FBS. The next day all wells were incubated in E2 free medium or $10^{-9} \mathrm{M} \mathrm{E2}$ medium for $0,2,4$ and 6 days. For drug sensitivity assay, 3000cells/well were plated in 96-well culture plates. The next day different concentrations of tamoxifen from $0-25 \mu \mathrm{M}$ were added into dilution medium for 72 hours. Before harvesting, $20 \mu \mathrm{l}$ MTT were added to each well. Cells were incubated for another 4 hours and dimethyl sulphoxide (DMSO, Sangon Biotech, Shanghai, China) were added $100 \mu l$ /well to dissolve the formazan after removing the culture medium. The plates were read at wavelength of $490 \mathrm{~nm}$ using Microplate Reader (Bio-Rad, Hercules, CA, USA). The culture medium was regarded as the bank control while the cells without reagents were the negative control.

\section{Clone formation for Cytotoxicity assay}

The drug sensitivity to tamoxifen was also measured by clone formation assay. Cells were plated in 6-well plates at a concentration of 500 cell /well. The next day different concentrations of tamoxifen from $0-500 \mathrm{nM}$ were added into $2.5 \mathrm{ml}$ dilution medium. The medium with drug was replaced every 5 days. After three weeks cells were fixed by methanol and stained with crystal violet. Clones containing over 50 cells were numbered under the microscope. 
Tumor formation in nude mice

Tumor xenografts were developed by injecting $5 \times 10^{6}$ cells in a Matrigel Basement Membrane Matrix subcutaneously into each flank of 4-5 weeks-old BALB/c nu/nu female mice and analyzed as described elsewhere with the following modifications. The estrogen groups were implanted subcutaneous in the neck region with a 60 day release $0.72 \mathrm{mg}$ estradiol pellet (Innovative Research of America) and the minus estrogen groups were implanted with placebo pellet during the entire experiment. Tumor volume was calculated every 5 days. All the investigations have been approved by the local ethical committee.

\section{Analysis of apoptosis by Flow Cytometry}

Cells with or without treatment were detected by flow cytometry using the Annexin V-FITC Apoptosis Detection Kit (BD Bioscience, San Jose, CA, USA). The experiments were performed according to the protocol described. Cells were trypsinized, collected and washed twice with ice-cold PBS at the indicated time. Then $1 \times 10^{5}$ cells were resuspended in $100 \mu \mathrm{l} 1 \times$ binding buffer, added with $5 \mu \mathrm{l}$ of annexin V-FITC and $5 \mu \mathrm{l}$ of PI following incubation for $15 \mathrm{~min}$ at room temperature in dark. Finally $400 \mu \mathrm{l} 1 \times$ binding buffer was added to each sample. The samples were analyzed by flow cytometry (Becton Dickinson, Franklin Lakes, NJ, USA). Within 1 hour using FACS calibur with Cell Quest software. Cells that stained FITC Annexin V but not PI were identified as apoptotic cells.

\section{Western Blot}

Cells were washed with ice-cold PBS and lysed with ice-cold RIPA buffer (Shennengbocai, Shanghai, China) ( $1 \times$ PBS, $1 \%$ NP40, $0.1 \%$ sodium dodecyl sulfate (SDS), 5mM EDTA, $0.5 \%$ sodium deoxycholate) containing protein inhibitor cocktail (Sigma, St. Louis, MO, USA) and phosphatase inhibitor sodium fluoride (NaF) and sodium vanadate (NaVO3). Proteins from each group were quantified by BCA Protein Assay Kit (Merck, Darmstadt, Germany) and separated by 5\%-10\% SDS-PAGE and transferred onto PVDF membrane (Millipore, Schwalbach, Germany). Membranes were blocked in 1×TBST with 5\% skim milk for 1 hour and subsequently incubated with the corresponding primary antibodies overnight at $4^{\circ} \mathrm{C}$. Then the membranes were washed three times with TBST and the incubated with secondary antibody for 2 hours at room temperature. After washing 3 times in TBST, each protein was detected using an ECL system (Merck, Darmstadt, Germany).

\section{SiRNA and transfection}

To knockdown the expression of PTEN in MCF7-3.1n cell line, we transfected the small interfering RNA (siRNA) of PTEN and Non-specific Negative control (NC, also obtained from Genepharma, Shanghai, China) with lipofectamine 2000 (Invitrogen) according to the manufacturer's protocol. Transiently transfected cells were harvested after 48 72h for MTT and protein analysis.

\section{Statistical analysis}

All experiments were repeated at least three independent times. All data were presented as means \pm SD of the mean and analyzed using SPSS 13.0 for Windows (SPSS Inc., Chicago, IL, USA). Differences between individual groups were analyzed by student $t$ test. $P$ values of $<0.05$ were considered statistically significant.

\section{Results}

Over-expression of MTDH in breast cancer cell line MCF-7

We detected the expression of MTDH in a series of breast cancer cells including ER $\alpha$ negative cell lines MDA-MB-231, MDA-MB-468 and ER $\alpha$ positive cell lines MCF7 and T47D (Fig. 1A). To determine whether MTDH played an important role in estrogen dependence and tamoxifen sensitivity in breast cancer, we designed MTDH over-expression vector $3.1 \mathrm{~m}$ to transfect MCF-7 cells and generated the MTDH over-expressed cells. The efficacy of MTDH expression in transfected MCF-7 cells was confirmed by RT-PCR on the RNA levels (Fig. 1B) while western blot on the protein levels (Fig. 1C). Cells transfected with $3.1 \mathrm{~m}$ showed significantly increased MTDH expression on both mRNA and protein levels compared to the control 3.1n. 
Fig. 1. Over-expression of MTDH in ER $\alpha$ positive breast cancer cells. (A) Relative MTDH mRNA levels in different cell lines. (B) Relative MTDH mRNA levels in $\mathrm{ER} \alpha$ positive breast cancer cell line MCF-7 transfected with 3.1-n and 3.1-m, respectively. Error bars represent means \pm SD of triplicate. (C) MTDH protein levels measured by western blot analysis.
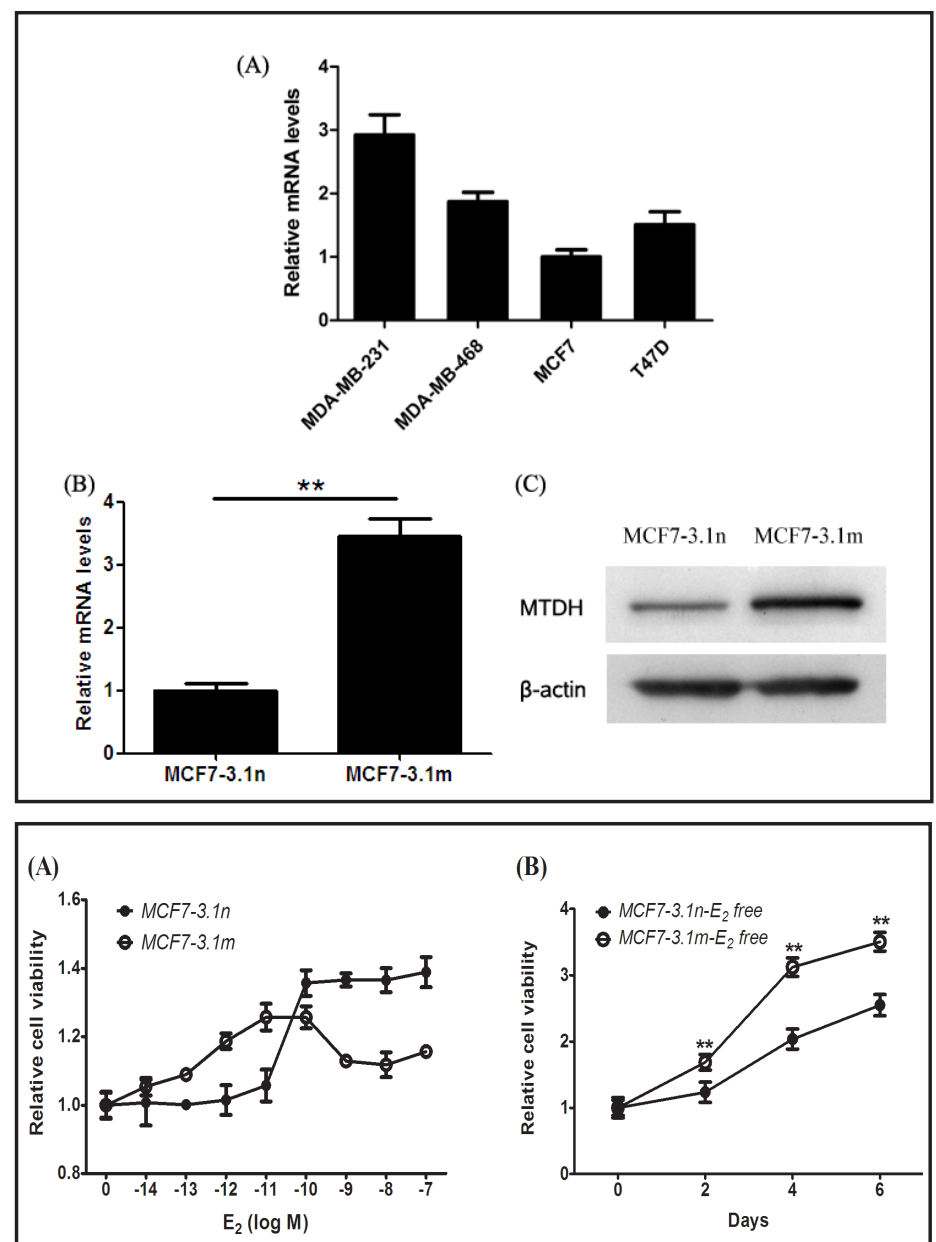
duced estrogen independent growth of cells (A) Different concentrations of $E_{2}$ were used to the cells for 6 days to test cells dose dependence to $\mathrm{E}_{2}$. The result showed the MTDH over-expression cells grew in low concentration of $\mathrm{E}_{2}$ at $10^{-13} \mathrm{M}$. While attenuated at concentration of E2 higher than $10^{-9} \mathrm{M}$. (B) and (C) Cells were treated with $10^{-9} \mathrm{M} \mathrm{E}_{2}$ medium or $E_{2}$ free medium to test the independence of $E_{2}$. At the absence of $E_{2}$, MTDH over-expression cells MCF$3.1 \mathrm{~m}$ promoted cell proliferation significantly contrast to the presence of $E_{2}$. (D) Graphs representing xenograft tumor volume at 42 days of at least five nude mice per group injected with MCF7-3.1n and MCF7-3.1m cells. $(\mathrm{p}<0.01)$ “*” means $\mathrm{p}<0.01$, “**” means $\mathrm{p}<0.05$ and " $\mathrm{N}$ " means there is no statistical significance between two groups.
(B)

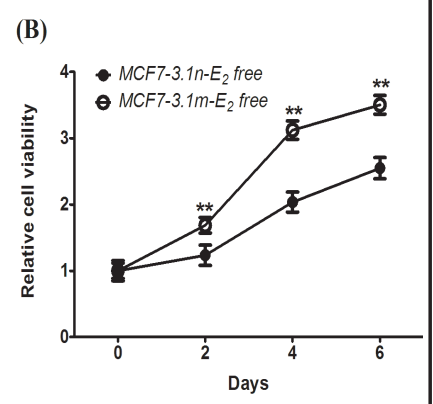

(D)
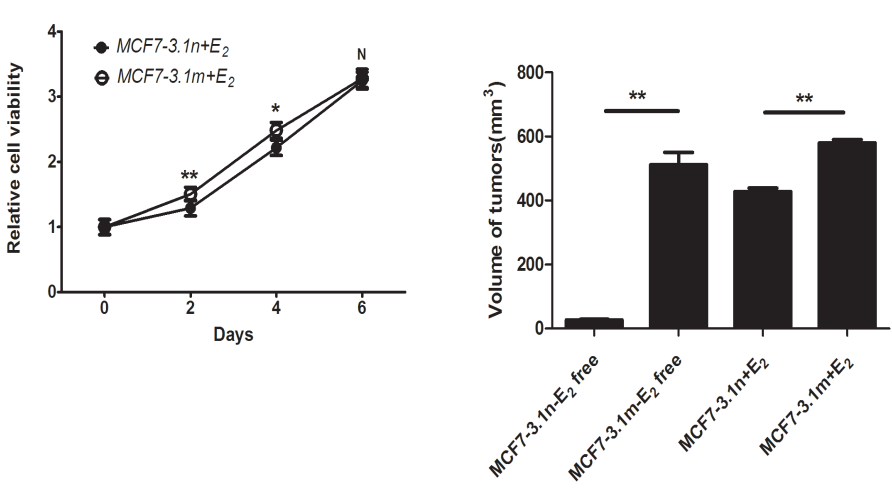

\section{MTDH induced estrogen independent growth of cells}

To demonstrate if MTDH induced MCF-7 cells are less dependent of estrogen, we treated cells with different concentrations of $\mathrm{E}_{2}$ for 96 hours and tested relative cell viability using cells in the phenol-free DMEM with CS-FBS as control. In contrast to MCF7-3.1n, the MTDH over-expressed cells are less dependent to $\mathrm{E}_{2}$ stimulation $(\mathrm{p}<0.001)$. The results showed that MCF7-3.1m cells could proliferate at the low dose of $\mathrm{E}_{2}$ of $10^{-13} \mathrm{M}$ contrast to that of $10^{-9} \mathrm{M}$ in MCF7-3.1n cells (Fig. 2A).

To verify our finding that MTDH made estrogen independent growth in MCF-7 cells, we treated the two stable selected cell lines with $\mathrm{E}_{2}$ at the concentration of $10^{-9} \mathrm{M}$ and the 


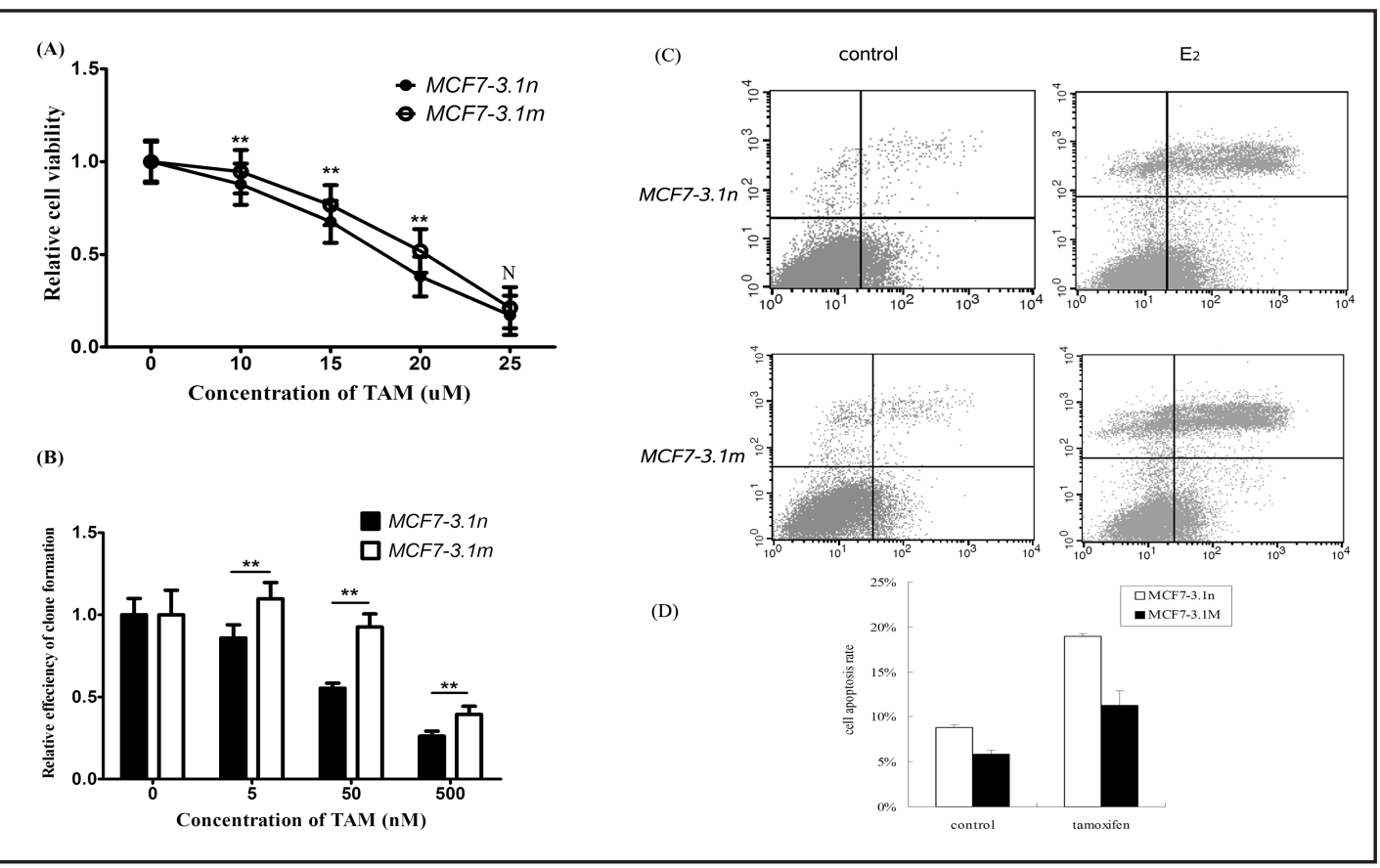

Fig. 3. MTDH increased resistance to tamoxifen in breast cancer cells. (A) Cytotoxicity was evaluated using the MTT assay. Cells were plated in 96-well culture plates at the concentration of 3000 cells/well. The next day different concentration of tamoxifen from 0-25uM were added into dilution medium. Cells were incubated for 72 hours. $(\mathrm{p}<0.01)$ (B) Clone formation assay. Marked difference of survival was observed between MCF7-3.1m and MCF7-3.1n cells. Data shown are the means \pm SD of three independent experiments $(\mathrm{p}<0.01)$. (C) and (D) MTDH inhibited cells apoptosis induced by tamoxifen. Cells with or without treatment of $10 \mathrm{uM}$ tamoxifen for 24 hours were detected by flow cytometry using the Annexin V-FITC Apoptosis Detection Kit (BD Bioscience, San Jose, CA, USA). The increased apoptosis rate induced by tamoxifen in MCF7$3.1 \mathrm{~m}$ was lower than that in MCF7-3.1n $(\mathrm{p}<0.01)$. “*” means $\mathrm{p}<0.01$, “**” means $\mathrm{p}<0.05$ and "N" means there is no statistical significance between two groups.

cells without $\mathrm{E}_{2}$ were used as control. The results showed that MTDH could promote cell proliferation with or without $\mathrm{E}_{2}$ (Fig. $2 \mathrm{~B}$ and $2 \mathrm{C}$ ). In the absence of $\mathrm{E}_{2}$, the increased growth rate of MCF7-3.1m cell lines was more significant than that of MCF7-3.1n cell lines (Fig. 2B). These findings indicated that over-expressed MTDH could promote estrogen-independent proliferation of MCF-7 cells.

To further explore the relationship between MTDH and estrogen independent growth in vivo, the xenograft model of human breast cancer cells in nude mice was adopted. Then we further verified above suggestions by using tumor formation in nude mice of the two stable selected cell lines with or without estradiol for 6 weeks. MCF7-3.1m could grow without E2 while little growth was seen in MCF7-3.1n (Fig. 2D). Therefore, we confirmed that MTDH could induce estrogen independent growth of MCF7 cells in vivo.

\section{MTDH induced cells resistance to tamoxifen}

As MTDH was indicated to play an important role in $E_{2}$ independent growth, we concluded that MTDH might regulate the sensitivity to the selective estrogen receptor modulator tamoxifen. To demonstrate it we used cell proliferation assay by MTT and clone formation in different concentrations of tamoxifen to measure cells sensitivity to tamoxifen in MCF7-3.1m and MCF7-3.1n. The results showed that the cells over-expressing MTDH were more resistant to tamoxifen ( $\mathrm{p}=0.009$ ) (Fig. 3A and 3B).

We further demonstrated the resistance induced by MTDH using testing apoptosis rate. After $24 \mathrm{~h}$ treated with tamoxifen at the concentration of $10 \mu \mathrm{M}$ to MCF7-3.1n and MCF7- 
Fig. 4. MTDH mediated estrogen-independent growth and tamoxifen resistance by regulating PTEN/PI3K/AKT pathway. (A)MTDH down regulated PTEN while increased the expression of pAKT and BCL2 by western blot. (B) and (C) MCF7-3.1n cells were transient transfected with the small interfering RNA (siRNA) of PTEN and NC. (B) Western blot analysis of pAKT, PTEN, BCL2. Suppression of PTEN induced up-regulation of pAKT (C) MTT assay for tamoxifen sensitivity. Suppression of PTEN induced tamoxifen resistance $(\mathrm{p}<0.01)$.

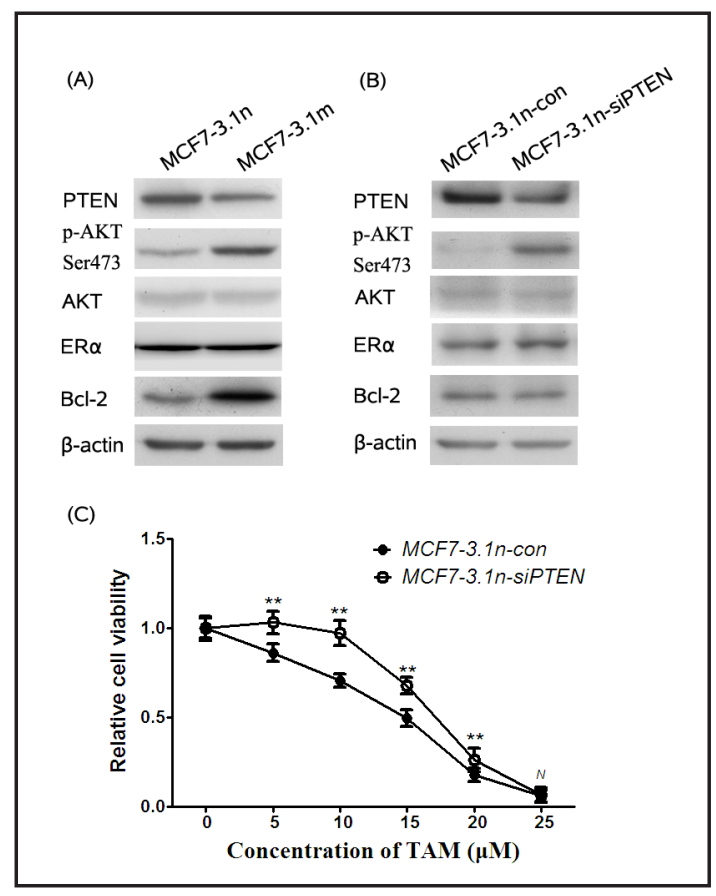

$3.1 \mathrm{~m}$ cells, the apoptosis rates were tested using the untreated cells as control. The results showed the apoptosis rates of MCF7-3.1m cells were lower than that of MCF7-3.1n treated with tamoxifen $(\mathrm{p}=0.005)$ (Fig. 3C and 3D). These indicated that MTDH could inhibit cell apoptosis induced by tamoxifen.

MTDH mediated estrogen-independent growth and tamoxifen resistance by regulating PTEN/PI3K/AKT pathway

The crosstalk of ER-E2 and non-endocrine signals are often reported to induce tamoxifen resistance $[13,14]$. Evidence indicated that estrogen independent growth and tamoxifen resistance are often associated with down-regulation of ER $\alpha$ [15]. We examined $E R \alpha$ expression and found that MCF-3.1m cells showed the same level of ER $\alpha$ as MCF-3.1n cells. Next, we determined the status of phosphorylated AKT (p-AKT, Ser473) because AKT activation has been implicated in tamoxifen resistance [16]. The results above showed that MTDH could increase expression of p-AKT and BCL2 which might contribute to tamoxifen resistance directly (Fig. 4A).

Phosphoinositide-3 kinase (PI3K)/AKT is a key factor in the mitogen-mediated cell growth pathway. Many factors are known to regulate p-AKT. The most direct and important upstream factor is PTEN (phosphatase and tensin homolog), a well known inhibitor of phosphoinositide-3 kinase. Inhibition of PTEN would increase the expression of p-AKT. Our results showed that MTDH over-expression down-regulated PTEN expression by western blot analysis (Fig. 4A).

To clarify the mechanism that MTDH mediated resistance to tamoxifen, we further silencing the expression of PTEN in MCF7-3.1n cells. Then MTT assay and western blot were all performed with cells transfected with siRNA of PTEN (MCF7-3.1n-siPTEN) and its negative control (MCF7-3.1n-con). Knockdown of PTEN resulted in un-sensitivity to tamoxifen in MCF7-3.1n-siPTEN cells. Results of western blot exhibited that the p-AKT levels was up-regulated (Fig. 4B). So we concluded that MTDH could lead to tamoxifen-resistance by suppression of PTEN.

We also confirmed the role of PTEN in regulation of resistance to tamoxifen. After silencing the expression of PTEN, we repeated the MTT assay to test the cell sensitivity to tamoxifen. The results indicated that when PTEN was down-regulated, MCF7-3.1n-siPTEN cells were more resistant to tamoxifen than the control cells (Fig. 4C). All the results above 
showed that MTDH mediated estrogen-independent growth and tamoxifen resistance by regulating PTEN/PI3K/AKT pathway.

\section{Discussion}

Breast cancer is the most common malignant tumor diagnosed in women worldwide and has become leading cause of cancer death in economically developing countries [1]. Tamoxifen, which can target ER $\alpha$ and block the action of estrogen on breast cancer, is still a first-line endocrine therapy for the management of all stages of ER $\alpha$-positive breast cancer. Five years of tamoxifen use was considered the standard duration, however, 10 years treatment is recommended recently. It can reduce the risk of recurrence by $41 \%$ on average in patients with $\mathrm{ER} \alpha$-positive tumors $[17,18]$. It was improved to greatly prolong disease-free survival and induce remission in over half of patients with ER-positive breast cancer [19]. Unfortunately, almost $50 \%$ of patients with advanced disease do not respond to first line treatment with tamoxifen. Furthermore, a significant percentage of patients experience tamoxifen resistance relapse, despite an initial positive drug response [18, 20]. The mechanisms of tumor resistance to tamoxifen therapy continue to pose a significant challenge to both clinicians and researchers. It might reside in the expression of specific molecules involved in different signaling pathways, which eventually could be used as predictive biomarkers of resistance. Moreover, these markers may be used to select patients might benefit from additional targeted treatments aside from ER $\alpha[21,22]$.

As found in 2002, MTDH has been found over-expressed in multiple cancers such as esophageal squamous cell carcinoma, breast carcinoma, melanoma, hepatocellular carcinoma, epithelial ovarian cancer and so on [23-25]. Many experiments have been done to demonstrate MTDH as an oncogene to regulate multiple molecular and pathways including PI3K/AKT pathway, NF-kappaB pathway, MAPK pathway, Wnt pathway, vascular endothelial growth factor (VEGF), transcription factor family (such as FOX01, FOXO3a) and so on to promote cell proliferation, migration, tumor metastasis and angiogenesis in various tumors $[7,25-30]$. In breast cancer our previous studies have shown that MTDH was over-expressed in more than $40 \%$ of the tumors which could indicate poor clinical outcomes [7, 9] and MTDH could enhance the invasiveness of cells by inducing epithelial to mesenchymal transition (EMT) [8]. Besides these we found that MTDH could induce multiple drug resistance in breast cancer such as doxorubicin, paclitaxel and cisplatin [31]. However there is still little report about the role of MTDH in estrogen dependence and tamoxifen resistance. In this study we found the functions of MTDH to promote estrogen independent growth and induce tamoxifen resistance.

Estrogen $\left(E_{2}\right)$ is demonstrated to be an endogenous factor which plays a critical role in normal mammary functions [32]. Studies in human breast cancer cells subjected to long-term estrogen deprivation (LTED) have demonstrated that these cells first developed hypersensitivity to low-dose estrogens and then became estrogen independent. This phenomenon seemed to contribute to resistance to endocrine therapy, which might in part result by increased levels of ER $\alpha$ and up-regulation of the MAPK, PI-3-kinase and mTOR growth factor pathways [33]. In our studies we first found that the oncogene MTDH could promote MCF-7 cells more sensitivity to $\mathrm{E}_{2}$ stimulating. Our results showed the two cell lines, MTDH over-expressed cells MCF7-3.1m and the control cells MCF7-3.1n, responded to $\mathrm{E}_{2}$ stimulation. The MCF7-3.1m cells could respond to $E_{2}$ at a very low dose of $10^{-13} \mathrm{M}$. MTDH could promote cell proliferation in MCF-7 much more obviously than control cell lines in $\mathrm{E}_{2}$ free medium. These results indicated that MTDH might induce estrogen independent growth. We further demonstrated this by xenograft tumor formation in nude mice. Tumors were smaller in nude mice injected with MCF7-3.1n cells without estrogen pellet while significantly larger with MCF7-3.1m cells. Our studies suggested MTDH could promote MCF-7 cells estrogen independent growth. Studies have indicated that ER positive breast cancer cells with estrogen independent growth were resistance to tamoxifen therapy [34, 
35]. We further tested the sensitivity to tamoxifen treatment of these two cell lines MCF73.1n and F7-3.1 $\mathrm{m}$ by MTT assay. We found that MTDH over-expressed cell lines expressed resistance to tamoxifen treatment contrast to control cell lines. Tumor cell apoptosis plays an important role in both preclinical and clinical responses to tamoxifen and tumor cell evasion of apoptosis contributes to tamoxifen resistance [36, 37]. To determine whether MTDH promoted tamoxifen resistance though reducing the apoptosis after treated with tamoxifen, we found that the increased apoptosis rate induced by tamoxifen in MCF7-3.1m was lower than that in MCF7-3.1n.

As down-regulation of $E R \alpha$ is often associated with estrogen independent growth and tamoxifen resistance, we examined ER $\alpha$ expression. As shown in Fig. 4, MCF7-3.1m cells revealed little decrease at the level of ER $\alpha$ than MCF7-3.1n cells, suggesting that MTDH impacted tamoxifen sensitivity though other signaling pathway. Next we determined the status of phosphorylated Akt (p-AKT) because Akt activation has been implicated in estrogen independent growth and tamoxifen resistance [16]. Western blot analysis revealed that MCF7-3.1m cells expressed high level of p-AKT than MCF7-3.1n cells.

Phosphoinositide-3 kinase /Akt is a key factor in the cell growth and proliferation pathways which regulated by many factors. PTEN (phosphatase and tensin homolog) , a well known inhibitor of phosphoinositide-3 kinase, is the most important and direct upstream factor. Reduction of its activity or suppression of PTEN expression would increase p-Akt levels. Down-regulation of PTEN expression in ER $\alpha$-positive tumors is associated with failure to tamoxifen treatment [38]. Our study found that MTDH over-expression down-regulated PTEN expression by 38\%. This suggested that MTDH induced tamoxifen resistance through inhibiting PTEN expression and activation of p-AKT. To further clarify the mechanism that MTDH mediate resistance to tamoxifen, we silenced the expression of PTEN in MCF-3.1n cells. The results showed that silencing of PTEN resulted in p-AKT over-expression by western blot and tamoxifen resistance by MTT assay. We failed to detect altered ER $\alpha$ and BCL-2 expression in response to suppression of PTEN, which suggests MTDH contributes to tamoxifen resistance through multiple mechanisms.

Based on our study, MTDH could mediate estrogen-independent growth and induce resistance to tamoxifen in ER $\alpha$-positive breast cancer cells. MTDH could reduce the expression of PTEN, up-regulate AKT and BCL2 and inhibit the apoptosis induced by tamoxifen. PTEN contributes to MTDH induced tamoxifen resistance in breast cancer, however, MTDH contributes to tamoxifen resistance through multiple mechanisms. Our findings provided the key functions of MTDH in tamoxifen resistance of breast cancer and indicated that MTDH could be a convincing target to reverse the resistance to tamoxifen in ER $\alpha$-positive breast cancer patients.

\section{Disclosure Statement}

Authors declare no conflict of interest.

\section{Acknowledgements}

This work was supported by National Natural Science Foundation of China (No. 30772133; No. 81072150; No. 81172529; No. 81272903) and Shandong Science and Technology Development Plan (No. 2012GZC22115).

\section{Reference}

$\rightarrow 1$ Jemal A, Bray F, Center MM, Ferlay J, Ward E, Forman D: Global cancer statistics. CA Cancer J Clin 2011;61:69-90. 


\section{Cellular Physiology $\quad$ Cell Physiol Biochem 2014;33:1557-1567 and Biochemistry \\ Xu et al.: MTDH Mediates Tamoxifen Resistance in MCF-7 Cells}

2 Fisher B, Costantino JP, Wickerham DL, Redmond CK, Kavanah M, Cronin WM, Vogel V, Robidoux A, Dimitrov N, Atkins J, Daly M, Wieand S, Tan-Chiu E, Ford L, Wolmark N: Tamoxifen for prevention of breast cancer: Report of the national surgical adjuvant breast and bowel project p-1 study. J Natl Cancer Inst 1998;90:1371-1388.

3 Tamoxifen for early breast cancer: An overview of the randomised trials. Early breast cancer trialists' collaborative group. Lancet 1998;351:1451-1467.

4 Massarweh S, Schiff R: Unraveling the mechanisms of endocrine resistance in breast cancer: New therapeutic opportunities. Clin Cancer Res 2007;13:1950-1954.

-5 Ward A, Balwierz A, Zhang JD, Kublbeck M, Pawitan Y, Hielscher T, Wiemann S, Sahin O: Re-expression of microrna-375 reverses both tamoxifen resistance and accompanying emt-like properties in breast cancer. Oncogene 2013;32:1173-1182.

6 Leszczyniecka M, Kang DC, Sarkar D, Su ZZ, Holmes M, Valerie K, Fisher PB: Identification and cloning of human polynucleotide phosphorylase, hpnpase old-35, in the context of terminal differentiation and cellular senescence. Proc Natl Acad Sci U S A 2002;99:16636-16641.

7 Hu G, Chong RA, Yang Q, Wei Y, Blanco MA, Li F, Reiss M, Au JL, Haffty BG, Kang Y: Mtdh activation by 8q22 genomic gain promotes chemoresistance and metastasis of poor-prognosis breast cancer. Cancer Cell 2009;15:9-20.

-8 Li X, Kong X, Huo Q, Guo H, Yan S, Yuan C, Moran MS, Shao C, Yang Q: Metadherin enhances the invasiveness of breast cancer cells by inducing epithelial to mesenchymal transition. Cancer Sci 2011;102:1151-1157.

-9 Su P, Zhang Q, Yang Q: Immunohistochemical analysis of metadherin in proliferative and cancerous breast tissue. Diagn Pathol 2010;5:38.

10 Zhao Y, Moran MS, Yang Q, Liu Q, Yuan C, Hong S, Kong B: Metadherin regulates radioresistance in cervical cancer cells. Oncol Rep 2012;27:1520-1526.

-11 Tokunaga E, Nakashima Y, Yamashita N, Hisamatsu Y, Okada S, Akiyoshi S, Aishima S, Kitao H, Morita M, Maehara Y: Overexpression of metadherin/mtdh is associated with an aggressive phenotype and a poor prognosis in invasive breast cancer. Breast Cancer 2014;21:341-349.

-12 Ao A, Morrison BJ, Wang H, Lopez JA, Reynolds BA, Lu J: Response of estrogen receptor-positive breast cancer tumorspheres to antiestrogen treatments. PLoS One 2011;6:e18810.

13 Bratton MR, Duong BN, Elliott S, Weldon CB, Beckman BS, McLachlan JA, Burow ME: Regulation of eralphamediated transcription of bcl-2 by pi3k-akt crosstalk: Implications for breast cancer cell survival. Int J Oncol 2010;37:541-550.

14 Nicholson RI, Hutcheson IR, Knowlden JM, Jones HE, Harper ME, Jordan N, Hiscox SE, Barrow D, Gee JM: Nonendocrine pathways and endocrine resistance: Observations with antiestrogens and signal transduction inhibitors in combination. Clin Cancer Res 2004;10:346S-354S.

15 Lykkesfeldt AE: Mechanisms of tamoxifen resistance in the treatment of advanced breast cancer. Acta Oncol 1996;35:S9-14.

-16 Kirkegaard T, Witton CJ, McGlynn LM, Tovey SM, Dunne B, Lyon A, Bartlett JM: Akt activation predicts outcome in breast cancer patients treated with tamoxifen. J Pathol 2005;207:139-146.

-17 Effects of chemotherapy and hormonal therapy for early breast cancer on recurrence and 15-year survival: An overview of the randomised trials. Lancet 2005;365:1687-1717.

18 Gradishar WJ: Tamoxifen--what next? Oncologist 2004;9:378-384.

19 Lewis-Wambi JS, Jordan VC: Treatment of postmenopausal breast cancer with selective estrogen receptor modulators (serms). Breast Dis 2005;24:93-105.

20 Ring A, Dowsett M: Mechanisms of tamoxifen resistance. Endocr Relat Cancer 2004;11:643-658.

21 Beelen K, Zwart W, Linn SC: Can predictive biomarkers in breast cancer guide adjuvant endocrine therapy? Nat Rev Clin Oncol 2012;9:529-541.

22 Hortobagyi GN: Toward individualized breast cancer therapy: Translating biological concepts to the bedside. Oncologist 2012;17:577-584.

23 Li C, Liu J, Lu R, Yu G, Wang X, Zhao Y, Song H, Lin P, Sun X, Yu X, Zhang Y, Ning X, Geng J: Aeg -1 overexpression: A novel indicator for peritoneal dissemination and lymph node metastasis in epithelial ovarian cancers. Int J Gynecol Cancer 2011;21:602-608. 


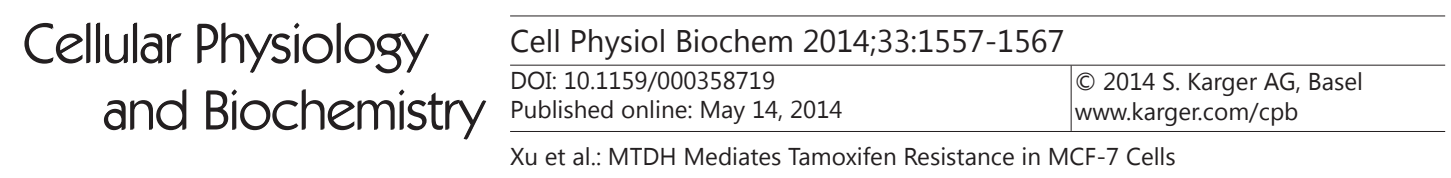

24 Yu C, Chen K, Zheng H, Guo X, Jia W, Li M, Zeng M, Li J, Song L: Overexpression of astrocyte elevated gene1 (aeg-1) is associated with esophageal squamous cell carcinoma (escc) progression and pathogenesis. Carcinogenesis 2009;30:894-901.

-25 Yoo BK, Emdad L, Su ZZ, Villanueva A, Chiang DY, Mukhopadhyay ND, Mills AS, Waxman S, Fisher RA, Llovet JM, Fisher PB, Sarkar D: Astrocyte elevated gene-1 regulates hepatocellular carcinoma development and progression. J Clin Invest 2009;119:465-477.

-26 Emdad L, Lee SG, Su ZZ, Jeon HY, Boukerche H, Sarkar D, Fisher PB: Astrocyte elevated gene-1 (aeg-1) functions as an oncogene and regulates angiogenesis. Proc Natl Acad Sci U S A 2009;106:21300-21305.

27 Song L, Li W, Zhang H, Liao W, Dai T, Yu C, Ding X, Zhang L, Li J: Over-expression of aeg-1 significantly associates with tumour aggressiveness and poor prognosis in human non-small cell lung cancer. J Pathol 2009;219:317-326.

28 Li C, Li R, Song H, Wang D, Feng T, Yu X, Zhao Y, Liu J, Wang Y, Geng J: Significance of aeg-1 expression in correlation with vegf, microvessel density and clinicopathological characteristics in triple-negative breast cancer. J Surg Oncol 2011;103:184-192.

29 Kikuno N, Shiina H, Urakami S, Kawamoto K, Hirata H, Tanaka Y, Place RF, Pookot D, Majid S, Igawa M, Dahiya R: Knockdown of astrocyte-elevated gene-1 inhibits prostate cancer progression through upregulation of foxo3a activity. Oncogene 2007;26:7647-7655.

30 Li J, Yang L, Song L, Xiong H, Wang L, Yan X, Yuan J, Wu J, Li M: Astrocyte elevated gene-1 is a proliferation promoter in breast cancer via suppressing transcriptional factor foxo1. Oncogene 2009;28:3188-3196.

31 Khuda, II, Koide N, Noman AS, Dagvadorj J, Tumurkhuu G, Naiki Y, Komatsu T, Yoshida T, Yokochi T: Astrocyte elevated gene-1 (aeg-1) is induced by lipopolysaccharide as toll-like receptor 4 (tlr4) ligand and regulates tlr4 signalling. Immunology 2009;128:e700-706.

-32 Imagawa W, Pedchenko VK, Helber J, Zhang H: Hormone/growth factor interactions mediating epithelial/ stromal communication in mammary gland development and carcinogenesis. J Steroid Biochem Mol Biol 2002;80:213-230.

-33 Santen RJ, Song RX, Zhang Z, Kumar R, Jeng MH, Masamura A, Lawrence J, Jr., Berstein L, Yue W: Long-term estradiol deprivation in breast cancer cells up-regulates growth factor signaling and enhances estrogen sensitivity. Endocr Relat Cancer 2005;12:S61-73.

-34 Cittelly DM, Das PM, Salvo VA, Fonseca JP, Burow ME, Jones FE: Oncogenic her2\{delta\}16 suppresses mir-15a/16 and deregulates bcl-2 to promote endocrine resistance of breast tumors. Carcinogenesis 2010;31:2049-2057.

35 Sachdeva M, Wu H, Ru P, Hwang L, Trieu V, Mo YY: Microrna-101-mediated akt activation and estrogenindependent growth. Oncogene 2011;30:822-831.

36 Mandlekar S, Kong AN: Mechanisms of tamoxifen-induced apoptosis. Apoptosis 2001;6:469-477.

-37 Naresh A, Thor AD, Edgerton SM, Torkko KC, Kumar R, Jones FE: The her4/4icd estrogen receptor coactivator and bh3-only protein is an effector of tamoxifen-induced apoptosis. Cancer Res 2008;68:63876395.

-38 Shoman N, Klassen S, McFadden A, Bickis MG, Torlakovic E, Chibbar R: Reduced pten expression predicts relapse in patients with breast carcinoma treated by tamoxifen. Mod Pathol 2005;18:250-259. 